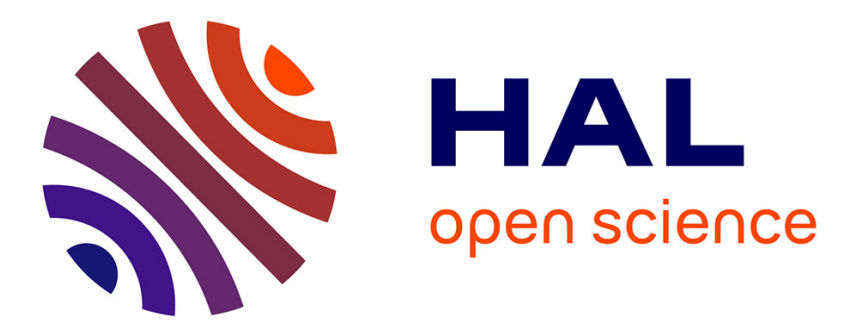

\title{
Détection de sources sous-marines et précision de l'impluvium par mesure des variations de salinité. L'exemple de la source de Cabbé-Massolins (Roquebrune-Cap-Martin, France) Eric Gilli
}

\section{To cite this version:}

Eric Gilli. Détection de sources sous-marines et précision de l'impluvium par mesure des variations de salinité. L'exemple de la source de Cabbé-Massolins (Roquebrune-Cap-Martin, France). Comptes Rendus de l'Academie des Sciences Serie IIa, 1999, 329, pp.109-116. hal-00748042

\section{HAL Id: hal-00748042 \\ https://hal.science/hal-00748042}

Submitted on 3 Nov 2012

HAL is a multi-disciplinary open access archive for the deposit and dissemination of scientific research documents, whether they are published or not. The documents may come from teaching and research institutions in France or abroad, or from public or private research centers.
L'archive ouverte pluridisciplinaire $\mathbf{H A L}$, est destinée au dépôt et à la diffusion de documents scientifiques de niveau recherche, publiés ou non, émanant des établissements d'enseignement et de recherche français ou étrangers, des laboratoires publics ou privés. 


\title{
Détection de sources sous-marines et précision de l'impluvium par mesure des variations de salinité. L'exemple de la source de Cabbé- Massolins (Roquebrune-Cap-Martin, France)
}

Detection of submarine springs and precision of the impluvium by measurement of variations of salinity, Example of the Cabbé-Massolins spring (Roquebrune-Cap-Martin, France)

\section{Eric Gilli*}

Centre d'étude du Karst (CEK), 1 3, rue Masséna, 06000 Nice, France (Reçu le 30 mars 1999, accepté le 12 avril 1999)

\begin{abstract}
Salinity measurements were used to detect and study submarine springs on the coast of southeastern France. Among them, the Cabbé-Massolins spring drains karstic formations in Arc de Nice (Alpes Maritimes, France). A salinity probe was placed in a spring. A comparison between the salinity variations and rainfall recorded at different meteorological stations, made it possible to define the location of the impluvium of the springs. C Académie des sciences / Elsevier, Paris.)
\end{abstract}

basin / karst / rainfall / s@ty / spring / submarine / France

Résumé - Une campagne de mesures de salinité en mer, le long du littoral niçois, a permis la détection de plusieurs sources sous-marines. Parmi elles, le groupe de Cabbé-Massolins correspond au drainage en mer d'unités karstiques mal connues, appartenant à l'arc de Nice (Alpes Maritimes, France). Une sonde autonome d'acquisition de salinité a été placée dans un des griffons de cet ensemble. Les variations de salinité à l'exutoire, comparées à la pluviosité des différentes stations météorologiques, permettent de préciser l'impluvium de ce groupe. (D Académie des sciences / Elsevier, Paris.)

impluvium / karst / pluviométrie / salinité / source / sous-marin / France

Note présentée par Chislain de Marsily. 


\section{Abridged version}

\section{Presentation}

In its eastern part, the Alpes-Maritimes district (France) contains several limestone mounts that are oriented norlhsouth. These units, that form an important watershed, are drained towards the south to karstic springs that are now under the sea on account of the versilian transgression.

Several submarine springs had previously been detected between Nice and Menton (Gloux, 1973; Gilli, 1989; Mangan, 1989). A recent research campaign was developed, using sea salinity and température measurements to identify the location of these springs and to detect others (Gilli, 1997).

\section{Detection and inventory of the springs}

A waterproof température and salinity probe SEABIRD SBE 19 was towed by a boat along the French coast between Nice and Menton. It was connected to the following equipment:

- DELL 386 notebook

- Garmin 45 GPS

- SEABIRD NMEA connexion box.

The full system is able to acquire thousands measurements pet day. For each point, salinity, température, latitude and longitude were measured. Several température and salinity anomalies were detected and then checked by scuba diving. Most of them were submarine springs (figure 1).

Three of them have a total discharge of 500 L-s-': Mortola near the Italian border, Cabbé in Cap-Martin and Pissarelles in Eze.

\section{Study of the Cabbé spring}

The group of Cabbé is made up of several springs placed along the north-south contact between the jurassic limestone of the Cap-Martin and the Cretaceous marls of Roquebrune bay. The total discharge is around $200 \mathrm{~L}-$ $\mathrm{s}-1$, the biggest spring is the Cabbé Massolins one. The water flows from a 25-m-long submarine cave (figure 2).

The watershed of this karstic aquifer is not well known. It is evaluated at $20 \mathrm{~km}$, but a structural analysis does not provide enough information to precisely identify the karstic units that participate in the feeding of the spring (figure 3).

A waterproof salinity probe was placed in the cave in order to study the salinity and the temperature of the spring for 15 days. The curves show steady variations caused by the tide effects and important ones caused by the discharge variations of the spring (figure 4). When the spring flow increases, the salinity and the température decrease. This characteristic was used during a second experiment to ident@ the watershed of the Cabbé group. The salinity probe was placed for another long period in the spring, between 20 December 1995 and 25 February 1996. The obtained curves were compared with rainfall data from several areas to research an eventual correlation between the variations of discharge at the spring and the rainy episodes on different parts of the supposed impluvium: La Turbie, L'Escarène, Sospel and Col de la Madone (Peille). The curves of La Turbie and L'Escarene give poor results (figure@, but the salinity and température curves are quite well correlated with the rainfall in the Col de la Madone and Sospel areas Cffigure 6). The effects of the rainfall are visible at the spring after a period of 8 to 9 days. The large différence for the last rainfall (1 to 7 February) is attributed to a cold period during which these areas received snow instead of tain. The effects at the spring were only visible after the snowmelt.

\section{Conclusions}

The use of a salinity and température probe makes possible the détection of submarine karstic springs and also gives information on their watersheds.

As it is difficult to install equipment to measure the discharge of these springs, the study of the salinity is a cheap way to get data on waterflow variations. 


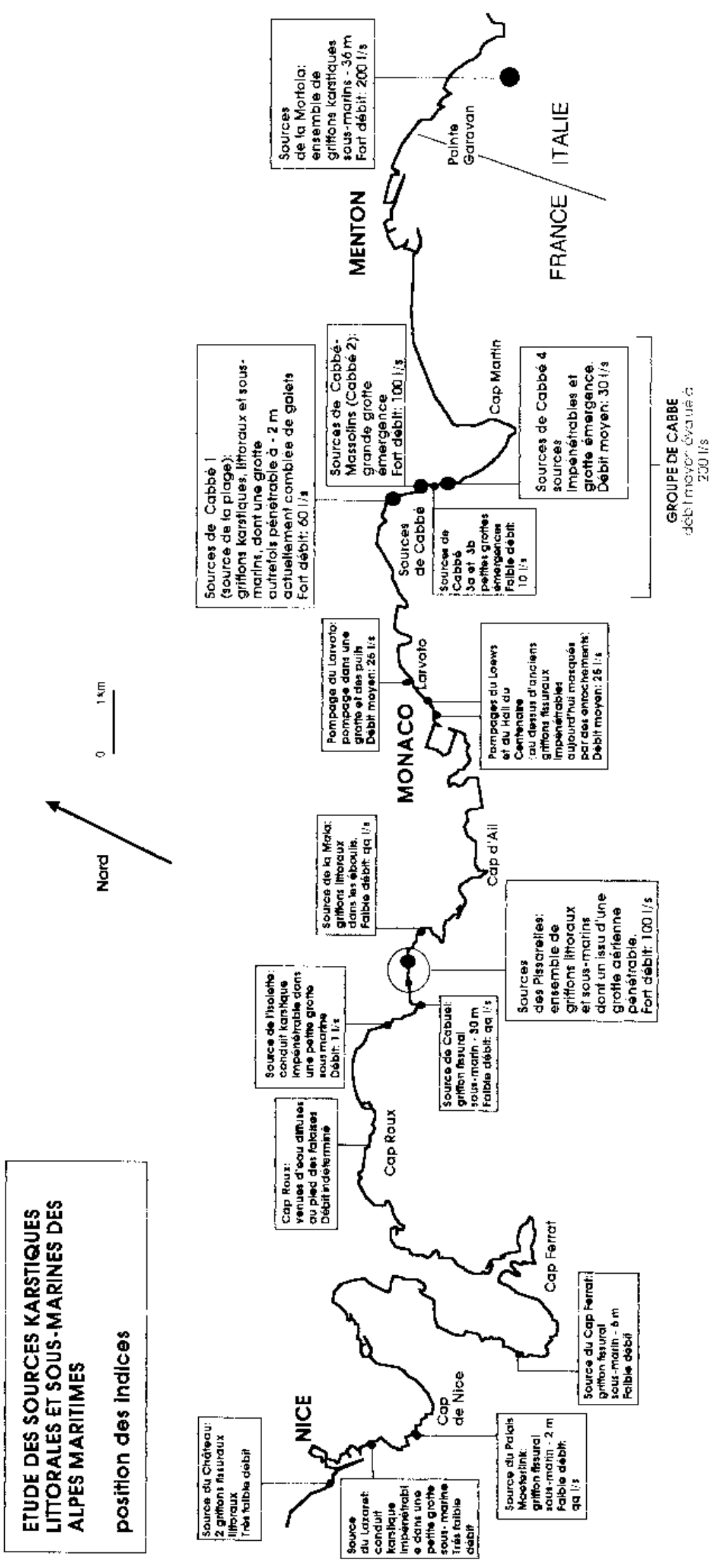




\section{Présentation}

Dans sa partie est, le département des Alpes-Maritimes comprend une série de chaînons calcaires orientés nord sud. Ces chaînons appartiennent à l'arc de Nice, sous unité des chaînes subalpines, dont la mise en place résulte de la surrection du massif cristallin de l'ArgenteraMercantour et du décollement de sa couverture sédimentaire. Cet ensemble, qui constitue un impluvium important, est principalement drainé vers le sud, en direction d'exutoires karstiques, actuellement submergés sous l'effet de la transgression versilienne. Plusieurs sorties d'eau douce sous-marines ont ainsi été décrites entre Nice et Menton (Gloux, 1973 ; Gilli, 1989 ; Mangan, 1989). Une campagne de recherches sur les émergences littorales et sous-marines des Alpes-Maritimes, financée par le ministère de l'Environnement (Diren-Paca) a été effectuée à l'aide d'un dispositif de mesure en continu de la salinité et de la température de l'eau de mer (Gilli, 1997).

\section{Détection et inventaire des émergences}

\section{1. Équipement embarqué}

Les mesures de salinité ont été réalisées à l'aide du dispositif suivant, embarqué sur une vedette :

- $\quad$ sonde étanche de température et conductivité Seabird SBE 19,

- ordinateur portable Dell 386,

- GPS Garmin 45,

- bottier de connections NMEA Seabird.

La sonde mesure la température et la salinité de l'eau, avec un pas de temps défini au préalable (en général, 1 mesure par seconde). La position du bateau, donnée par le GPS (Global positioning system) est enregistrée en continu. Il a ainsi été possible de mesurer plusieurs milliers de points par journée de travail. La sonde était fixée à un flotteur, à $30 \mathrm{~cm}$ sous la surface de la mer, loin des éventuelles sources de contamination (échappement, circuit de refroidissement, brassage dû à l'hélice). Les anomalies détectées (chute de la température et de la salinité) ont ensuite été contrôlées en plongée. Les débits ont été évalués en mesurant la vitesse d'un nuage de colorant injecté dans les conduits karstiques.

\subsection{Précision du dispositif}

La sonde utilisée a permis la détection des rejets des douches des plages publiques, ainsi que des eaux usées des bateaux au mouillage. Elle présente donc une précision tout à fait satisfaisante, compte tenu des objectifs de l'étude. Les sources déjà connues ont parfaitement été identifiées par le dispositif.

Bien qu'une erreur systématique variable soit introduite par l'armée américaine dans le signal GPS, la précision du positionnement est de l'ordre de $20 \mathrm{~m}$, ce qui est suffisant pour cette étude.

\subsection{Résultat des recherches en mer}

La plupart des anomalies contrôlées en plongée correspondaient bien à des venues d'eaux karstiques, dont plusieurs étaient inconnues à ce jour. Un inventaire et une carte des sorties principales ont été réalisés (figure 1). Trois sites importants totalisent $500 \mathrm{~L} . \mathrm{s}^{-1}$ : La Mortola à 
la frontière franco-italienne, Cabbé au cap Martin et les Pissarelles à Eze. Une source, le Larvoto (50 L.s $\left.{ }^{-1}\right)$, est déjà exploitée par Monaco.

Le site de Cabbé, sur le flanc ouest du cap Martin (Roquebrune, Alpes-Maritimes) a fait l'objet d'une étude spécifique.

\section{Le site de Cabbé}

\subsection{Description des sources de Cabbé}

Le groupe de Cabbé est un delta de griffons, dont le débit total est estimé à environ 200 L.s ${ }^{-1}$. Les points de sortie d'eau sont alignés selon un axe nord-sud, le long du contact redressé, entre le Jurassique calcaire du cap Martin et le Crétacé marneux de la baie de Roquebrune. En amont de la zone d'émergence, ces terrains sont recouverts par un épais masque de poudingues miocènes.

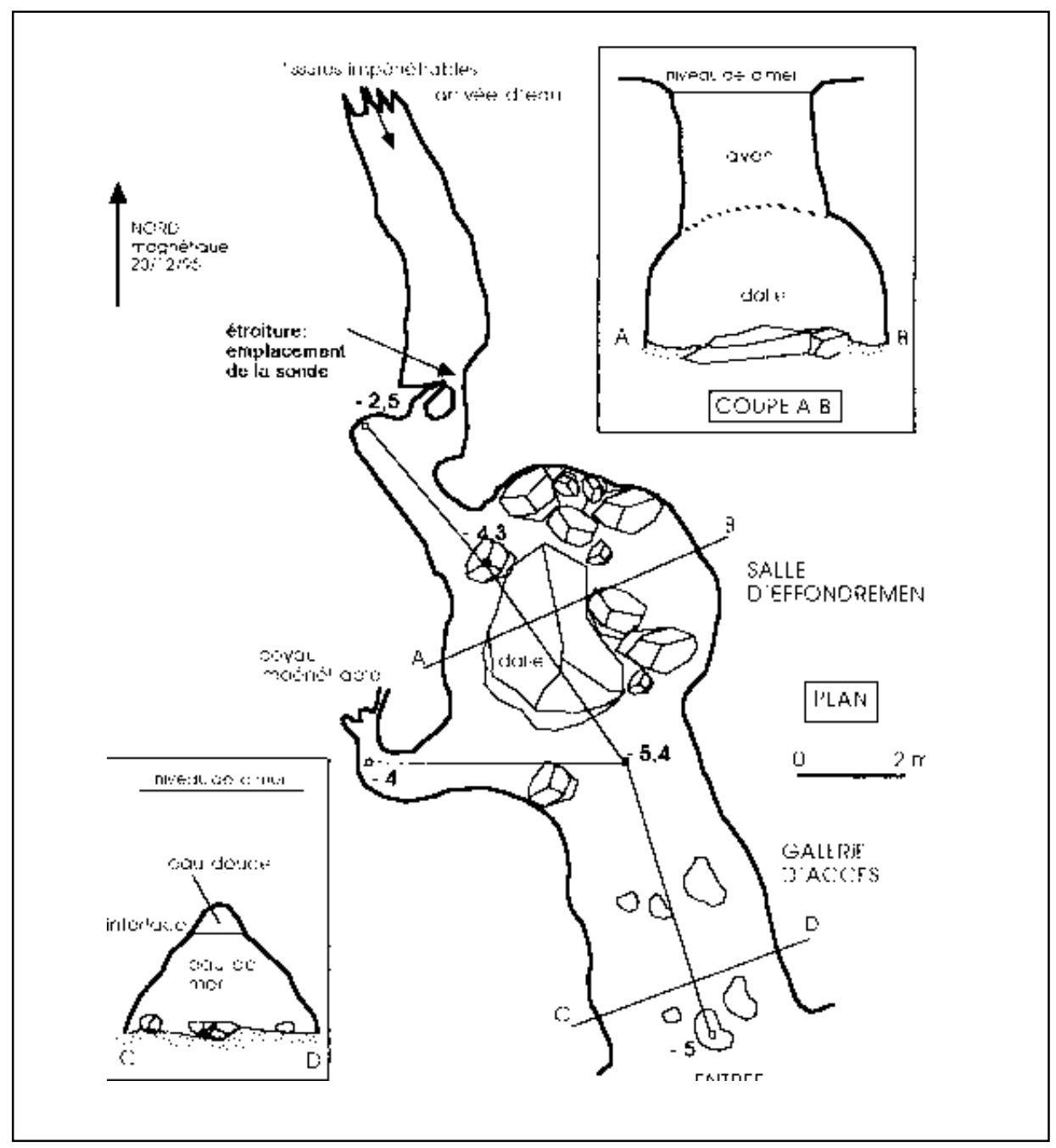

Figure 2. Plan de la grotte sous-marine de Cabbé-Massolins (Roquebrune, Alpes-Maritimes, France).

Map of Cabbé-Massolins submarine cave (Roquebrune, AlpesMaritimes, France). 
Le griffon principal est la source sous-marine de CabbéMassolins (figure 2), bien visible depuis le sentier littoral du cap Martin. Celle-ci sort d'une grotte totalement noyée, explorée sur environ $25 \mathrm{~m}$ de long. On entre par une galerie de section triangulaire de $4 \mathrm{~m}$ sur $3 \mathrm{~m}$, qui donne accès à un aven d'effondrement bien visible sur la plage rocheuse. Un passage au nordest permet d'atteindre une étroiture d'où provient le flux d'eau douce. Le franchissement de cette étroiture a permis de reconnaître environ $10 \mathrm{~m}$ de conduit karstique, avant de buter sur des fissures impénétrables par lesquelles arrive l'eau. Le débit est de $100 \mathrm{~L} . \mathrm{s}^{-1}$ en étiage. Cette cavité a été choisie pour réaliser un captage expérimental ; une étude spécifique a été menée sur le site.
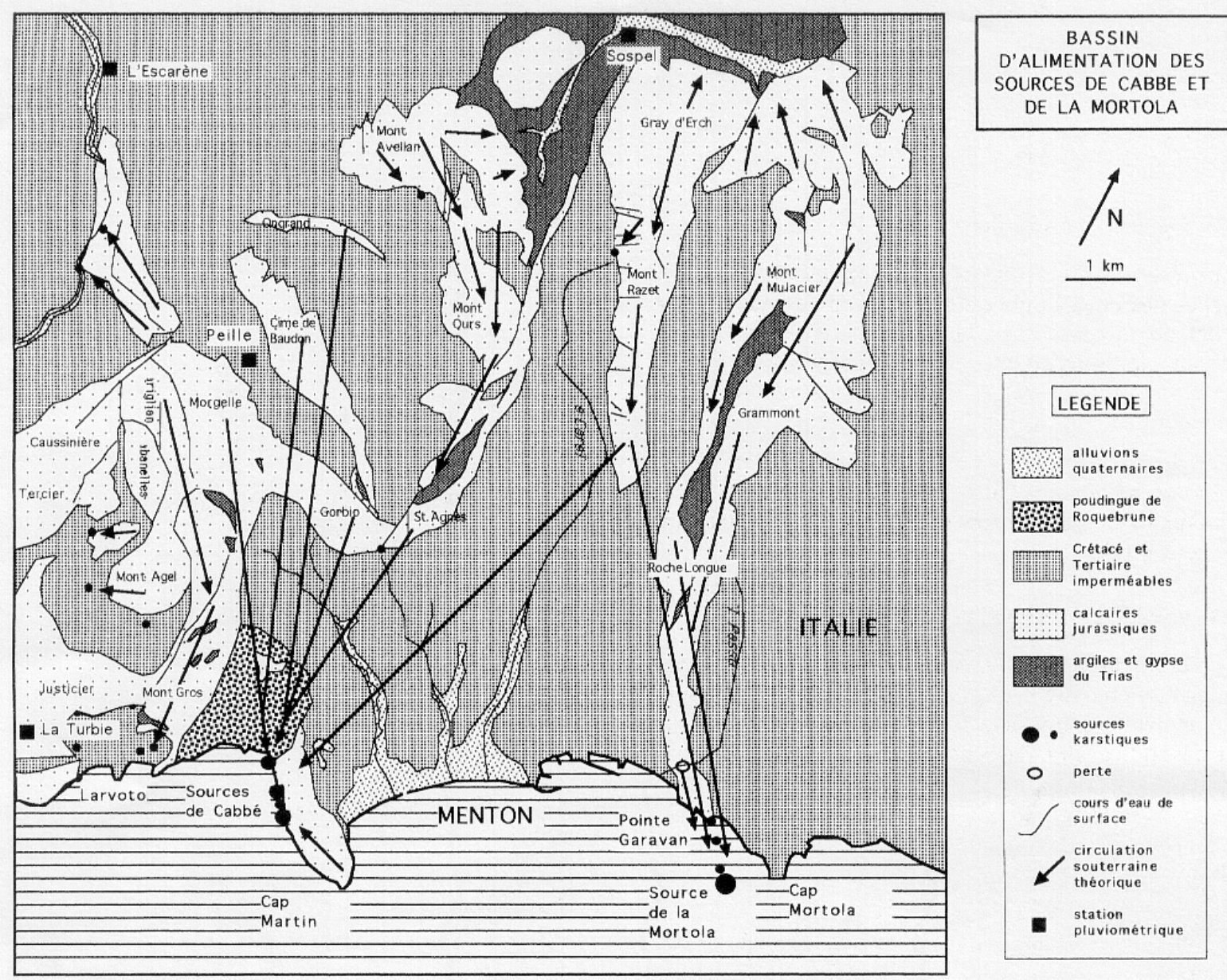

Figure 3 : Bassin d'alimentation des sources sous-marines de Cabbé et de la Mortola. Impluvium of the Cabbé and Mortola submarine springs 


\subsection{Bassin d'alimentation (figure 3)}

Le débit moyen est évalué à 200 L.s-', ce qui représente environ $20 \mathrm{~km}^{2}$ de surfaces karstifiées. Le bassin d'alimentation de la source reste à étudier. L'analyse structurale de ce secteur ne permet pas d'individualiser parfaitement les zones karstiques pouvant participer à l'alimentation de cet aquifère. Les différentes unités structurales de ce secteur, bien que très compartimentées par la tectonique, possèdent, en effet, de nombreux contacts calcaire/calcaire permettant la communication des aquifères karstiques. La plupart des émergences drainent donc plusieurs unités. Toutes les unités calcaires situées dans le quadrilatère, Monaco et Menton au sud, L'Escarène et Sospel au nord, peuvent théoriquement être retenues.

Pour la source de Cabbé, il est donc intéressant de vérifier si les chaînons calcaires situés à l'ouest (plateau du Justicier, mont des Mules) peuvent être inclus dans l'impluvium et si le bassin d'alimentation s'étend très au nord de la zone littorale.

\subsection{Caractéristiques physiques de la source}

Une sonde Seabird étanche permettant l'acquisition autonome de la température, de la pression etde la salinité a été placée durant l'automne 1995, pendant 15 jours, dans l'étroiture (figure 2), au niveau de l'interface eau douce/eau de mer.

L'acquisition des données s'est faite avec un pas de temps de 15 min. Les courbes obtenues (température, salinité et pression) (figure 4) montrent des variations importantes :

- la salinité varie de 5 g.L $\mathrm{L}^{-1}$ (eau saumâtre) à 37 g.L $\mathrm{L}^{-1}$ (eau de mer) ;

- la température passe de $17,5^{\circ} \mathrm{C}$ (eau du karst) à $22^{\circ} \mathrm{C}$

(eau de mer) ;

- le niveau de la mer oscille de $28 \mathrm{~cm}$.

La courbe de pression (profondeur) montre de nettes variations sinusoïdales, d'une amplitude de $28 \mathrm{~cm}$, avec une période d'environ $12 \mathrm{~h}$. La sonde étant fixe, ces variations sont liées aux variations de niveau de la mer, donc à la marée.

À marée montante, on note une augmentation de la salinité et de la température, le maximum correspondant au maximum de la marée. À marée descendante, le phénomène inverse s'observe. En moyenne, une variation de niveau de $25 \mathrm{~cm}$ entraîne une variation de salinité de 3 à 4 g.L $\mathrm{L}^{-1}$ et de température de $0,4{ }^{\circ} \mathrm{C}$.

Cela s'explique par une progression vers l'amont du biseau salé, lorsque le niveau marin augmente. La position de l'interface est donc fonction de la marée.

Indépendamment des variations liées à la marée, les courbes de salinité et température montrent des chutes brutales, attribuables à une augmentation du débit de la source. Le biseau salé est alors chassé vers l'aval. 


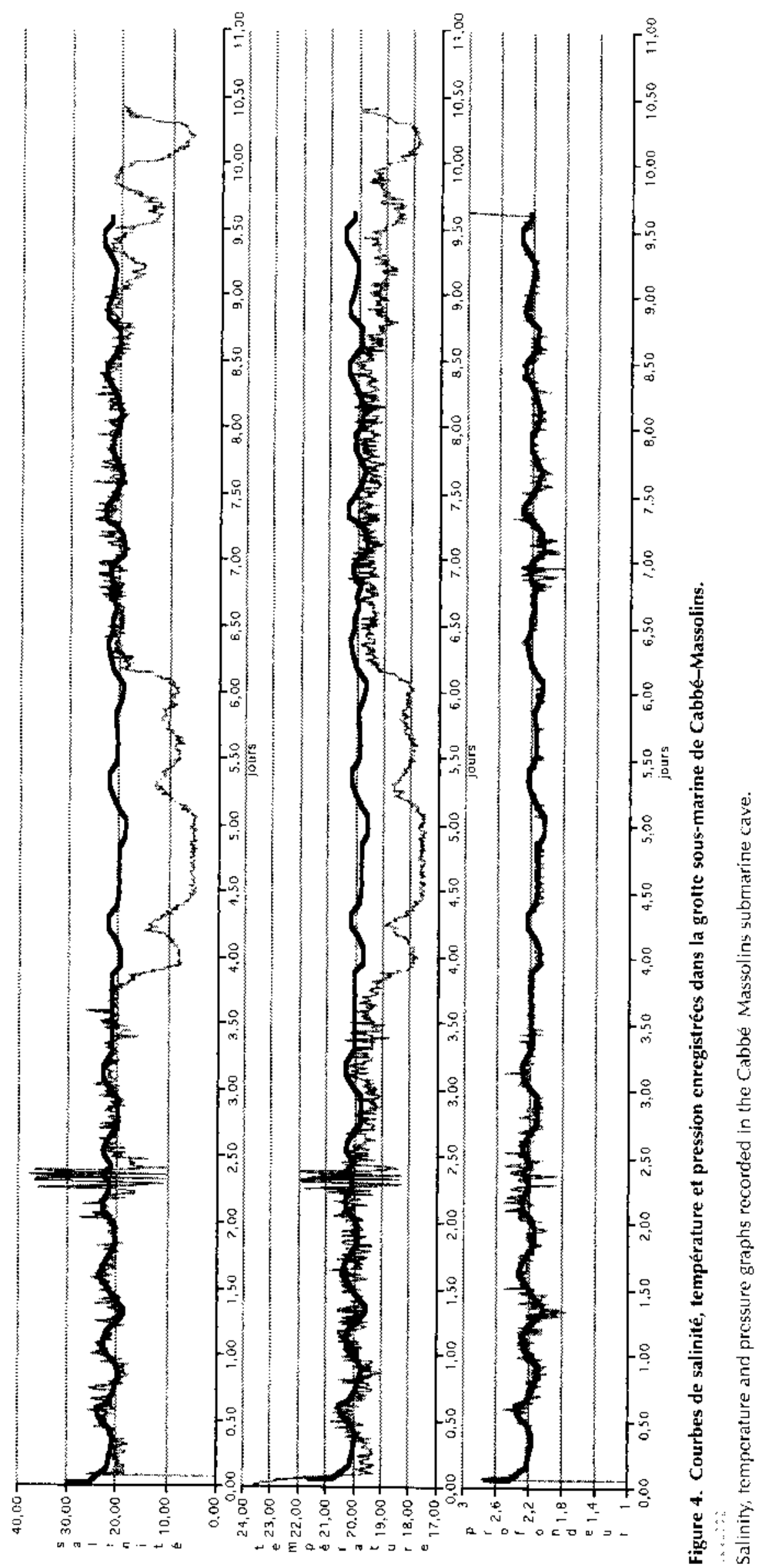


Figure 5. Essai de corrélation entre les variations de salinité de la source suus-marine de CabbeMassolins el les données pluviomóliriques du moyen-pays Niçois. Secteurs de la Turbie et L'Escartìnc.

Fxperirtent of correlation be Iweer salinity variations in the Cabbés Massolins submarine sprimg and the inland rairiall dala. Ares of la Turbies and l'Escanche:

Figure 6. Essai de corrélation entré les variations de salinité de la source de Cabbe-Massolins et les données pluviométriques du moyen-pays niçois. Secteurs de Peille (col de la Madone) et de Sospel.

Experiment of correlation be tween salinity variations in the Cabbe Massolins submarime spring and the inland rainfall dalia. Aread of Peide! lool de la Madonel and Sospel.
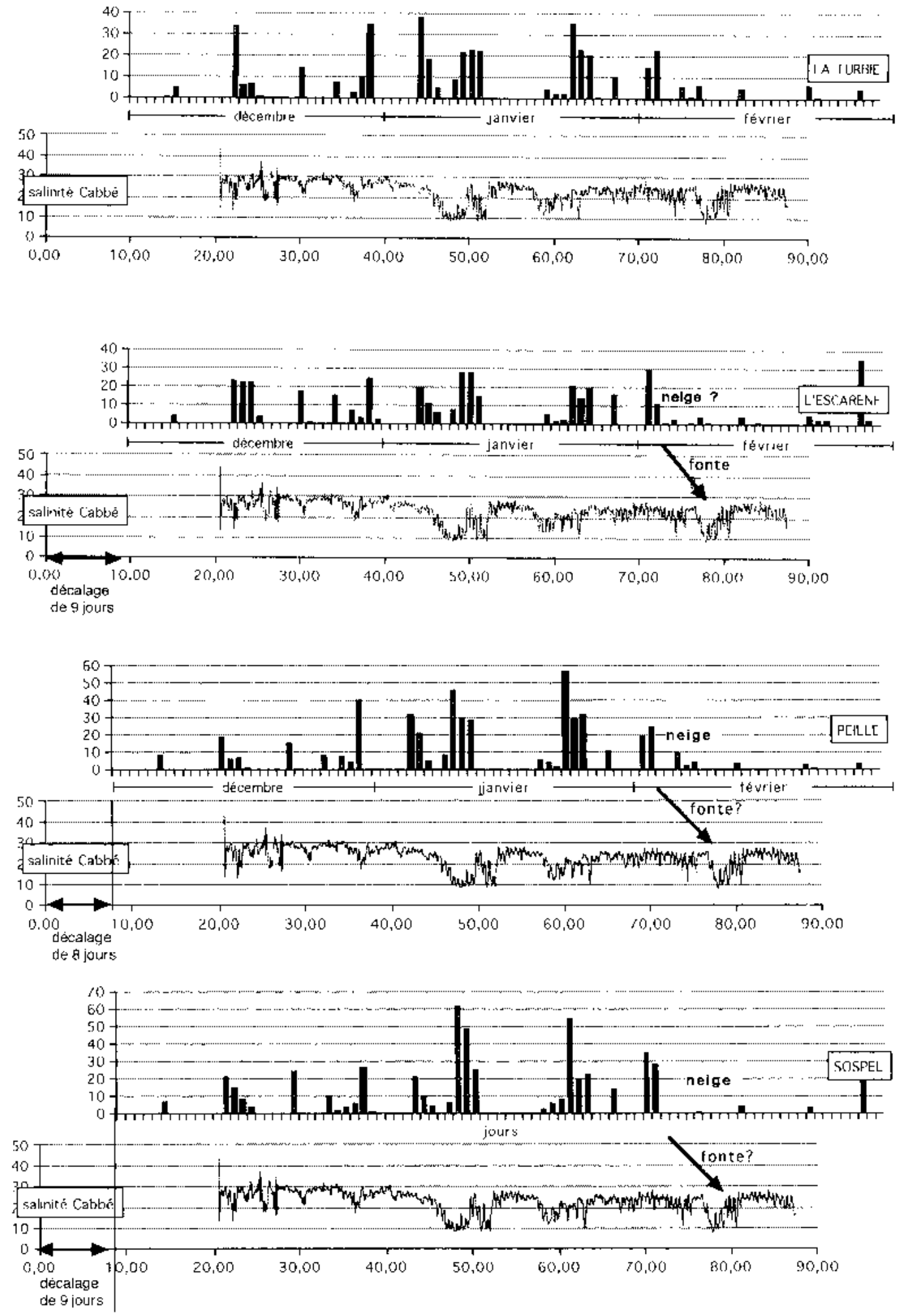


\subsection{Essai de définition du bassin d'alimentation}

La possibilité de suivre des variations nettes de salinité et température a été utilisée lors d'une deuxième expérience, pour préciser l'étendue du bassin versant. En effet, l'expérience précédente a montré que les variations de salinité au niveau de l'étroiture reflétaient les variations de débit. La sonde a été placée au même endroit, avec un pas de temps de $2 \mathrm{~h}$, du 20 décembre 1995 au 25 février 1996. Les courbes ainsi obtenues ont été ensuite comparées aux données pluviométriques de différents secteurs géographiques, au nord du cap Martin : la Turbie à l'est, le col de la Madone (Peille) et l'Escarène au nord, Sospel au nord-est, en vue de rechercher une éventuelle corrélation avec un ou plusieurs de ces secteurs. Les courbes ont donc été comparées en appliquant différents décalages, du fait du temps de transfert des ondes de crue. Les distances par rapport à la source sont de $6 \mathrm{~km}$ pour le col de la Madone, de $12 \mathrm{~km}$ pour Sospel et l'Escarène. Les pluies ayant affecté une grande superficie du département, les contrastes entre les différentes zones sont malheureusement peu marqués. Les zones de la Turbie et de J'Escarène offrent une corrélation médiocre (figure 5). Par contre, pour les secteurs du col de la Madone, et de Sospel (figure 6), en appliquant un décalage de 8 à 9 jours, on peut relier les 5 principaux épisodes pluvieux aux variations de salinité. Les points mesurés étant respectivement à 12 et $6 \mathrm{~km}$ de la source, on obtient des vitesses de réaction comprises entre 30 et $60 \mathrm{~m} \cdot \mathrm{h}^{-1}$. L'important décalage dans le temps de la dernière chute de salinité (du 15 au 20 février), par rapport aux dernières précipitations (du $1^{\text {er }}$ au 7 février) peut s'expliquer par un stockage de l'eau sous forme de neige, avec une fonte différée. Une vague de froid, avec des précipitations neigeuses, a en effet été observée à cette époque.

\subsection{Interprétation géologique}

L'impossibilité d'établir une corrélation nette avec la pluviosité de la Turbie permet a priori d'écarter les unités du justicier et du Mont Gros, pourtant proches du secteur étudié etde ne retenirque celles comprises entre Sospel et le col de la Madone à Peille (Gorbio, SainteAgnès, Baudon, Ongrand, le mont Ours, Avellan, et peut-être le Gray d'Erch et le mont Razet, bien que ces derniers soient plutôt supposés participer à l'alimentation de la source de la Mortola). Il n'y a malheureusement pas de station pluviométrique dans ce secteur.

\section{Conclusion}

Sous réserve de bonnes conditions de navigation, les mesures en continu de salinité et de température,couplées à l'usage du GPS, permettent de détecter facilement les émergences sous-marines.

Bien que réalisée sur une courte durée, et de ce fait très incomplète, l'expérience d'analyse des variations de salinité pour préciser les bassins d'alimentation des sources marines a donné d'intéressants résultats. Ces sources, lorsqu'elles sont totalement immergées, posent en effet des problèmes d'acquisition des débits et d'échantillonnage. L'appareil utilisé peut donc offrir une alternative peu coûteuse. Cette méthode ne remplace bien sûr pas les opérations de traçage, mais peut aider à leur mise en place. Il serait intéressant de pouvoir travailler sur des périodes plus longues et de disposer d'une densité de stations pluviométriques plus importante, afin d'obtenir un contraste de pluviosité mieux marqué entre les différentes zones. La fin de l'été, où l'on observe généralement de nombreux orages très localisés, nous semble la période la mieux adaptée. 


\section{Références}

Gilli E. 1989, De l'eau sous la mer, Monte Carlo Côte d'azur n 39

Gilli E. 1997. Etude des sources sous-marines et littorales de la partie est des Alpes maritimes. Rapp. int. Dir. Rég. Environn. Ministère de l'Environnement, Aix, 508 p., 56 fig., 30 profils, 5 cartes.

Gloux B. 1973. Les sources d'eau douce ou saumâtres sous-marines, Rapp. biblio. DEA, lab océano. Fac; Sc., Nice

Mangan Ch. 1989. Disponibilités en eau souterraine de la zone littorale entre Nice et menton (06), Rapp. int. Compagnie Générale des Eaux, Nice 